\title{
TERMINAL VELOCITIES FOR A LARGE SAMPLE OF O STARS, B SUPERGIANTS, AND WOLF-RAYET STARS
}

\author{
R. K. PRINJA, M. J. BARLOW \\ Department of Physics \& Astronomy \\ University College London \\ Gower Street, London WC1E 6BT \\ England
}

\author{
I. D. HOWARTH \\ JILA \\ University of Colorado \\ Boulder, Colorado \\ U.S.A.
}

\begin{abstract}
We argue that easily measured, reliable estimates of terminal velocities for early-type stars are provided (1) by the central velocity asymptotically approached by narrow absorption features in unsaturated UV P Cygni profiles, and (2) by the violet limit of zero residual intensity in saturated P Cygni profiles. We use these estimators and high resolution $I U E$ data to determine terminal velocities, $v_{\infty}$, for 1810 stars, 70 early B supergiants, and 35 Wolf-Rayet stars. For OB stars our values are typically $15-20 \%$ smaller than the extreme violet edge velocities, $v_{\text {edge }}$, while for WR stars $v_{\infty}=$ $0.76 v_{\text {edge }}$ on average. We give new mass-loss rates for WR stars which are thermal radio emitters, taking into account our new terminal velocities and recent revisions to estimates of distances and to the mean nuclear mass per electron. We examine the relationships between $v_{\infty}$, the surface escape velocities, and effective temperatures.
\end{abstract}

The full paper is due to be published in the 1990 October 1 issue of the Astrophysical Journal. 